\title{
Measuring the Complex Modulus of Asphalt Concrete Using Ultrasonic Testing
}

\author{
J. K. Van Velsor ${ }^{1}$, L. Premkumar ${ }^{2}$, G. Chehab ${ }^{3, *}$ and J.L. Rose ${ }^{4,5}$ \\ ${ }^{1}$ FBS, Inc., 143 Hawbaker Industrial Drive, Suite 102, State College, PA 16803 \\ ${ }^{2}$ Project Engineer, Applied Research Associates \\ ${ }^{3}$ Dep. of Civil and Environmental Engineering, American University of Beirut, PO Box 11-0236, Beirut, Lebanon 1107-2020 \\ ${ }^{4}$ Dep. of Engineering Science and Mechanics, The Pennsylvania State University, 212 Earth and Engineering Sciences Building, \\ University Park, PA 16802. \\ ${ }^{5}$ FBS, Inc., 143 Hawbaker Industrial Drive, Suite 102, State College, PA 16803
}

Received 23 February 2011; Revised 17 March 2011; Accepted 14 June 2011

\begin{abstract}
Dynamic modulus mastercurves are essential for the design and modeling of asphalt concrete (AC). One way of improving the accuracy of the upper asymptote of the mastercurve is to test at extremely high frequencies or extremely low temperatures. Ultrasound is used extensively in the nondestructive testing of materials and the work completed here demonstrates the potential for the application of this technology to AC. Since testing at extremely low temperatures is not practical, a new ultrasonic technique is developed for measuring the complex moduli of AC. A theoretical explanation of the measurement process is provided. Two AC specimens were tested using the ultrasonic method and the dynamic modulus method in the indirect tensile test (IDT) mode. Both test techniques were performed at four different temperatures. The mastercurves were constructed using time-temperature superposition on the IDT test data and the upper asymptotes were extrapolated. The ultrasonic data was shifted to the desired reference temperature and the predicted moduli were compared to those of the IDT test. It was found that the moduli predicted using the ultrasound measurement agreed well for the specimen with a lower air-void content and differed more for the specimen with a higher air-void content. The phase angles predicted by the ultrasonic method were higher than those obtained from the IDT test. It is believed that this was a result of wave scattering from air-voids and aggregates. Suggestions are made to further increase the accuracy of the technique.
\end{abstract}

Keywords: Asphalt Concrete, Ultrasound, IDT, Complex Modulus, Time-Temperature Superposition

\section{Introduction}

The ability to predict damage in asphalt concrete (AC) is highly dependent on the accuracy with which the material moduli can be measured. For this reason, efforts have been made to test $\mathrm{AC}$ materials at higher loading frequencies in order to obtain a more accurate upper asymptote of the dynamic modulus mastercurves. Recently, Kweon and Kim (1) introduced an impact resonance (IR) method for performing measurements in the sonic kilohertz range. They demonstrated the ability to construct the dynamic modulus and phase-angle mastercurves by performing IR measurements at different temperatures.

One of the primary objectives of the work introduced here was to further increase the accuracy of the AC mastercurves by extending material testing capabilities into the ultrasonic range. This would be a significant contribution, enabling practitioners to better characterize the dynamic modulus mastercurves used in the Mechanistic Empirical Pavement Design Guide (MEPDG) for pavement

\footnotetext{
*E-mail address: ghassan.chehab@aub.edu.lb

ISSN: 1791-2377 @ 2011 Kavala Institute of Technology. All rights reserved.
}

design and would also serve as an important tool for the improved modeling of AC.

Testing at ultrasonic frequencies is equivalent to testing at extremely low temperatures, using typical loading frequencies in the hertz range, with the added benefits of being nondestructive and not needing extremely cumbersome testing equipment. The ultrasonic method also has several benefits over the IR method. For example, the repeatability of the IR method is heavily dependent on the material, mass, shape, and impact velocity of the impactor, whereas with the ultrasonic method, the excitation characteristics are highly stable as calibrated ultrasonic transducers are used. Additionally, it is possible to measure the complex shear modulus using the ultrasonic method; an achievement much more difficult, albeit not impossible, using the IR method.

By measuring the density and longitudinal- and shearwave velocities, all other common engineering material properties (e.g. Poisson ratio, Lame' constant, elastic and shear moduli, etc.) can be obtained via simple algebraic relations. It is common to measure the properties of metals and other highly elastic materials in this way. While a notable amount of work has also been completed on the characterization of plastics and other viscoelastic materials, 
the amount of literature available regarding the ultrasonic characterization of $\mathrm{AC}$ is relatively sparse, most likely because the highly attenuative and inhomogeneous nature of these materials can impede wave propagation.

Sztukiewicz (2) correlated the measured longitudinalwave velocity to the bulk specific gravity of AC samples at different temperatures and ages. It was found that wave velocity increased with increasing bulk specific gravity and decreased with increasing temperature. The increase with bulk specific gravity is in agreement with that which is seen for elastic materials and the decrease with temperature can be attributed to the softening of the AC specimen at higher temperatures. It was also reported that decreased wave velocity can be correlated to aging of the AC. Stukiewicz attempted to use the longitudinal-wave velocity for the in situ characterization of AC, though, the proposed characterization parameters were of a highly empirical form and therefore it is not believed that the results are applicable to a wide variety of specimens.

Most recent attempts to use ultrasound as a characterization tool for AC were presented by Norambuena-Contreras (3) and by Pellinen and Witczak (4). Witczak et al. (5) evaluated several potential techniques for the development of a Simple Performance Test (SPT) for the characterization of $\mathrm{AC}$. One of the techniques proposed involved the measurement of ultrasonic longitudinal wave velocity in an AC specimen, which was then used to calculate the Dynamic Elastic Modulus. Their elastic modulus predictions were made using equations from the theory of elasticity and therefore did not account for the viscoelastic nature of the material (i.e. the predicted properties had no complex component). Pellinen and Witczak (4) did not present any acquired ultrasonic data and dismissed the ultrasonic technique as a viable candidate for a SPT.

\section{Methodology}

In this study, complex modulus tests were conducted on two AC specimens and dynamic modulus, dynamic shear modulus, and phase angle mastercurves were constructed using the time-temperature superposition principle. Ultrasonic longitudinal- and shear-wave data was collected on the same specimens. The ultrasonic data was plotted on the mastercurves and compared to the extrapolated highfrequency asymptote determined from the complex modulus tests.

The AC material used was a paver mixture obtained from the surface layer of Interstate 79 in Butler County, Pennsylvania. The surface mixture (A26 mixture) is a $9.5 \mathrm{~mm}$ with $2.2 \%$ baghouse fines and SBS Polymer modified binder with a grade of 76-22.

The specimens were compacted to a height of $115 \mathrm{~mm}$ at compaction temperature of $155^{\circ} \mathrm{C}\left(310^{\circ} \mathrm{F}\right)$ and later cored and cut to obtain $100 \mathrm{~mm} \times 38 \mathrm{~mm}$ (diameter $\times$ height) test specimens. The two specimens, henceforth referred to as Specimen I and Specimen II, were compacted to $2.2 \%$ and $3.5 \%$ air void content.

All the complex modulus testing was conducted using closed loop universal loading systems manufactured by MTS. Data acquisition was performed using a separate computer fitted with a National Instruments ${ }^{\circledR} 6329$ DAQ card and data acquisition programs prepared in Labview were used for data collection. The testing was conducted in the indirect tension mode (IDT) due to the specimen dimensions required for ultrasonic testing.

The deformation values were measured by means of Linear Variable Differential Transformers (LVDTs). For the IDT test, four X-SB LVDTs, with a range of $\pm 0.25 \mathrm{~mm}$ were used for measuring the vertical and horizontal deformations. The deformation values were measured along a gauge length of 38. $\mathrm{mm}$ for specimen diameter of $100 \mathrm{~mm}$ and a $6 \mathrm{in}$ Lottman Head with strip width of 0.77 in was used for setting up the specimen.

The complex modulus tests in IDT mode was conducted at $-20,-10,0$ and $+10^{\circ} \mathrm{C}$ at frequencies of $20,10,5,1$ and 0.5 Hz. The test involved applying a sinusoidal load to the specimen and obtaining the vertical and horizontal deformation response from two LVDTs mounted on each side of the specimen. The loads were applied to induce a maximum horizontal strain of 70 microstrains to ensure the specimen remained in the linear viscoelastic range. For data analysis, only the last six cycles were considered. Additional information on the testing is documented by Chehab et al. (6).

The viscoelastic solution developed by Momen (7) was used for computing the Dynamic modulus in the IDT mode and is shown in Eq. (1).

$$
\left|E^{*}\right|=\frac{2 * P_{0}}{\pi a d} \frac{\beta_{1} v_{2}-\beta_{2} v_{1}}{v_{2} V_{0}-\beta_{2} U_{0}}
$$

where $P_{0}$ is the applied load amplitude, $a$ is the strip width of the loading head, $d$ is the specimen diameter, $V_{0}$ is the amplitude of the vertical deformation, and $U_{0}$ is the amplitude of the horizontal deformation.

The Dynamic shear modulus was calculated using Eq. (2) and the Poisson's ratio was assumed to be 0.3 for the temperature ranges used.

$$
|G *|=\frac{|E *|}{2(1+v)}
$$

where $G^{*}$ is the complex shear modulus, $E^{*}$ is the complex modulus, and $v$ is Poisson's ratio.

All ultrasonic testing was completed with a standalone ultrasonic testing system with a $1000 \mathrm{~V}$ toneburst generator and $100 \mathrm{MHz}$ A/D converter. All longitudinal data was collected using $500 \mathrm{kHz}$ center-frequency transducers and all shear data was collected with $750 \mathrm{kHz}$ center-frequency transducers. A $0.50 \square s$ pulse width and $100 \mathrm{MHz}$ sampling frequency was used in all experiments. The very short pulse width was used in order to obtain as large an excitation bandwidth as possible.

\section{Linear Viscoelasticity And Time-Temperature Superposition}

Asphalt concrete is a viscoelastic material that exhibits both time and temperature dependence. In the linear viscoelastic range, or undamaged state, it is also thermorheologically simple and the effect of time and temperature can be expressed through one joint parameter (5). The process of combining the testing temperature and frequencies is possible through a variable termed 'Reduced Frequency', which is simply the product of frequency and a shift factor. 
The viscoelastic material property, as a function of frequency or time, can be shifted along the horizontal time axis to form a characteristic mastercurve at a desired reference temperature $(8)$.

\section{Complex Modulus}

A unit response function denotes the response of a linear viscoelastic material to a unit input function. Complex modulus $\left(E^{*}\right)$ is the unit response function of a sinusoidal input function. The dynamic modulus, which is the magnitude of the complex modulus, is equivalent to the strain response for a unit sinusoidal stress load. The dynamic modulus is calculated by dividing the steady state sinusoidal stress amplitude $\left(\square_{l}\right)$ by the steady state sinusoidal strain amplitude $\left(\square_{2}\right)$. The phase angle $(\phi)$ is related to the time lag, $\Delta t$, between the stress input and strain response and the frequency of testing.

Complex modulus is decomposed into two major components, the storage and loss modulus as represented in Eq. (3) and in Fig. (1).

$$
E^{*}=E^{\prime}+i E^{\prime \prime}
$$

where $E^{\prime}$ is the storage modulus, and $E^{\prime \prime}$ is the loss modulus. The dynamic modulus is the amplitude of the complex modulus and is defined as

$$
\left|E^{*}\right|=\sqrt{\left(E^{\prime}\right)^{2}+\left(E^{\prime \prime}\right)^{2}}
$$

The values of the storage and loss moduli are related to the dynamic modulus and phase angle by

$$
\begin{aligned}
& E^{\prime}=\left|E^{*}\right| \cos \varphi \\
& E^{\prime \prime}=\left|E^{*}\right| \sin \varphi
\end{aligned}
$$

From Fig. (1) it can be observed that the value of phase angle can vary between $0^{\circ}$ and $90^{\circ}$. If the phase angle is $0^{\circ}$, the material is completely elastic and if the phase angle is $90^{\circ}$, the material is completely viscous.

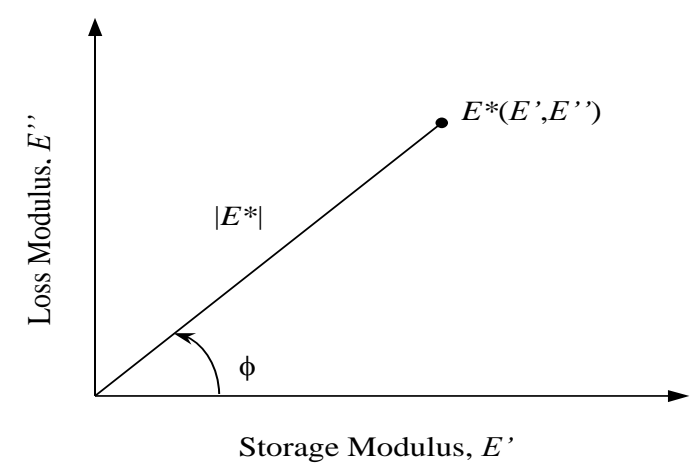

Fig. 1. Complex modulus decomposed into real and imaginary components.

\section{Ultrasonic Theory and Measurement}

The governing equations of linear viscoelasticity take on the same form as the governing equations of linear elasticity when integral transform methods are used. This analogy is often referred to as the elastic-viscoelastic correspondence principle and it has significant implications. Because the time-dependence of the stress-strain relations can be removed using integral transform methods, it is possible to obtain the transformed viscoelastic solution by solving the corresponding elastic problem with complex material properties. Read (9) was the first to utilize the Fourier integral transform for such purposes and Christensen (10) provides a detailed presentation of the correspondence principle using Laplace integral transforms. It is therefore possible to use the elastic wave equation, with complex material properties, to describe ultrasonic wave propagation in $\mathrm{AC}$.

To date, several ultrasonic velocity measurement techniques have been developed to predict the moduli of AC. One detail that all of these techniques have in common is that the predicted moduli are real numbers, as opposed to complex numbers that are characteristic of viscoelastic materials. It would be a valuable contribution to be able to calculate the complex moduli of AC from ultrasonic wave propagation measurements.

\section{Solution of the Complex Wave Equation}

In order to calculate the complex moduli of AC from wave propagation data, it is useful to examine the one-dimensional complex wave equation,

$$
\frac{d^{2} u}{d x^{2}}=\frac{1}{c^{*}(i \omega)^{2}} \frac{d^{2} u}{d t^{2}}
$$

where $u(x, t)$ is the particle displacement, $\square$ is the circular frequency, and $c^{*}(i \omega)$ is the complex frequencydependent phase velocity within the medium of interest. In infinite media the phase velocity is equivalent to the longitudinal or shear wave velocity. If the distance to the nearest specimen edge is much greater than the ultrasonic wavelength, the media is considered infinite.

The solution to Eq. (7) is the same as for the elastic wave equation (with the exception of the now complex circular wavenumber) and can be found in sources such as Christensen (10), Haddad (11), or Barshinger and Rose (12). The solution of Eq. (7) is of the form,

$u(x, t)=U_{0} e^{i\left(\omega t-k^{*} x\right)}=U_{0} e^{i\left(\omega t-\left(k^{\prime}+i k^{\prime \prime}\right) x\right)}=$
$U_{0} e^{k^{\prime \prime} x} e^{i\left(k^{\prime} x-\omega t\right)}=U_{0} e^{-a(\omega) x} e^{i\left(\frac{\omega}{c(\omega)} x-\omega \tau\right)}$

where $\alpha(\omega)$ is the frequency dependent attenuation constant which is responsible for the decay in wave amplitude with increasing propagation distance and is a direct consequence of the complex circular wavenumber 
$k^{*}$. The single-prime in Eq. (8) represents the real part of the circular wavenumber while the double-prime represents the complex part. The initial wave amplitude is given by $U_{0}$. It can be seen from the last expression in Eq. (8) that,

$k^{\prime}=\frac{\omega}{c(\omega)}=\operatorname{Re}\left(\frac{\omega}{c^{*}(\omega)}\right)$

$$
k^{\prime \prime}=-\alpha(\omega)=\operatorname{Im}\left(\frac{\omega}{c^{*}(\omega)}\right)
$$

Noting that the relationship between the phase velocity and circular wavenumber is of the form,

$$
c *(\omega)=\frac{\omega}{k *}
$$

an equation for the complex wave velocity can be developed from Eq. (9), Eq. (10), and Eq. (11),

$$
c^{*}(\omega)=\frac{1}{\frac{1}{c(\omega)}-i \frac{\alpha(\omega)}{\omega}}
$$

where $c(\omega)$ is the measured longitudinal or shear wave velocity within the material. Since the quantities $c(\omega)$ and $\alpha(\omega)$ are real, they can be directly measured by experiment. Therefore, with knowledge of the measurable wave velocity in the material and the rate of attenuation of the wave, it is possible to calculate the complex phase velocities from Eq. (12) and, in turn, the complex moduli. The complex Shear modulus can be calculated from,

$$
G^{*}=\rho \cdot c_{S}^{*}
$$

where $c_{S}^{*}$ is the complex shear wave velocity and $\rho$ is the material density. from,

The complex Young's Modulus can then be calculated

$$
E^{*}=\left[\frac{3-4\left(\frac{c_{S}^{*}}{c_{L}^{*}}\right)^{2}}{1-\left(\frac{c_{S}^{*}}{c_{L}^{*}}\right)^{2}}\right] G *
$$

This concludes the solution of the complex wave equation and derivation of the corresponding complex material and acoustic properties. The following two sections will deal with the measurement of the wave velocity and attenuation constant in an AC specimen.

\section{Wave Velocity Measurement}

In this study wave velocity was measured using a throughtransmission arrangement in which the ultrasound is transmitted, propagates directly through the specimen, and is received by a second transducer. This is shown Fig. (2). To obtain the most accurate velocity estimate possible, measurements were made for two different thicknesses of the same specimen and the propagation distance was taken to be the difference in axial length of the specimen thicknesses. In this way, any time delay introduced by the electronics is nullified. Data was collected on Specimen I at thicknesses of $h_{2}=38.1 \mathrm{~mm}$ and $h_{1}=34.38 \mathrm{~mm}$ and on Specimen II at thicknesses of $h_{2}=32.43 \mathrm{~mm}$ and $h_{2}=27.78 \mathrm{~mm}$.

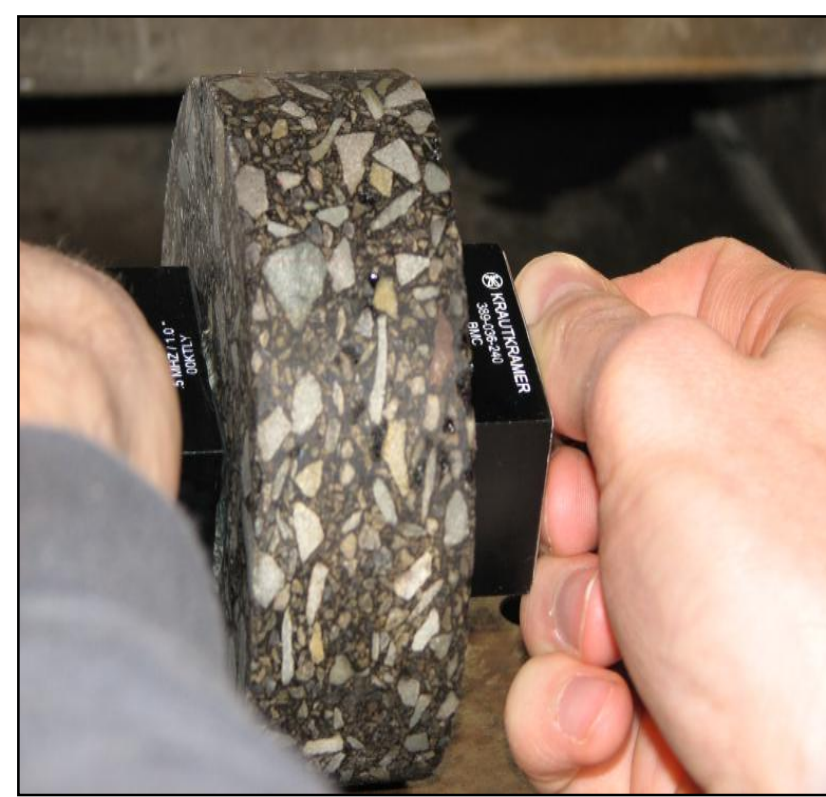

Fig. 2. Photograph showing the ultrasonic wave velocity and attenuation constant measurement.

All acquired ultrasonic signals were time-averaged 25 times to improve the signal-to-noise ratio. Spatial averaging was also employed in an attempt to account for the inhomogeneity of the specimens. Plots (a) and (b) in Fig. 3 show 10 time-averaged signals obtained at different locations on Specimen I using longitudinal and shear waves, respectively. Plots (c) and (d) in Fig. (3) are the spatially averaged results obtained by averaging the 10 signals in (a) and (b). The red line seen in Fig.(3) plots (c) and (d) represents the envelope of the wave packet and are useful for peak and wave-front location purposes. The arrival time of the wave is often referenced from the peak or wave front. 


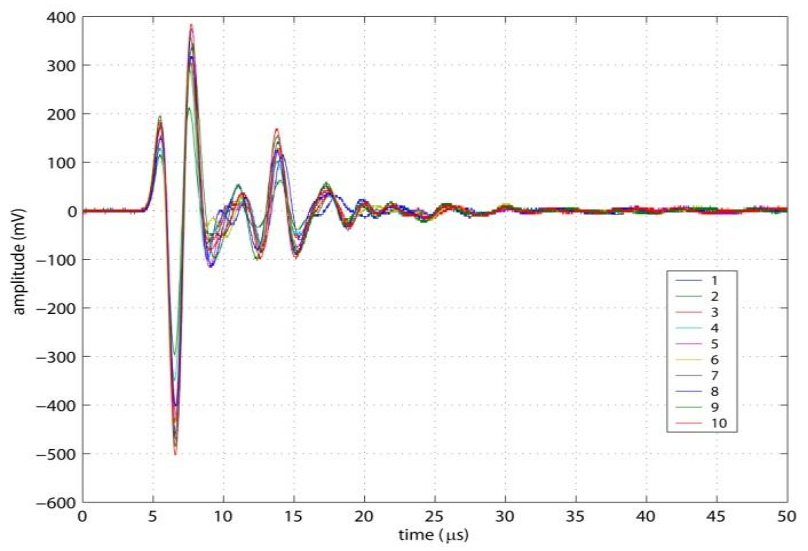

(a) Time-Averaged Longitudinal Waveforms

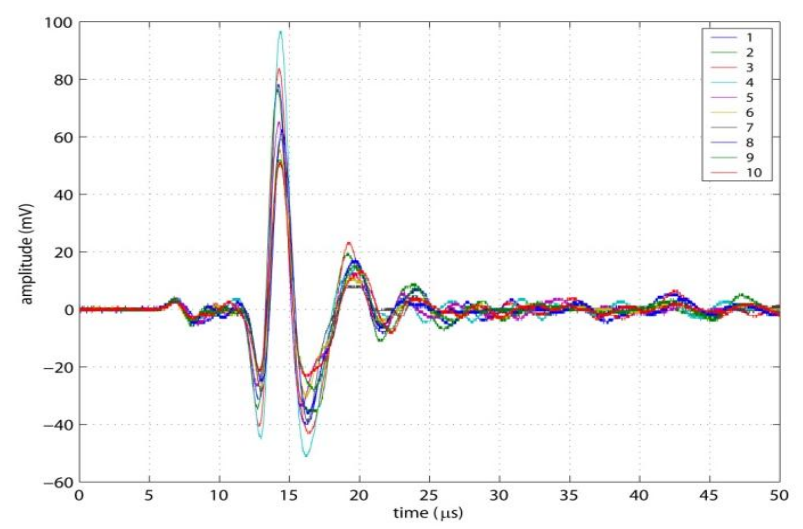

(b) Time-Averaged Shear Waveforms

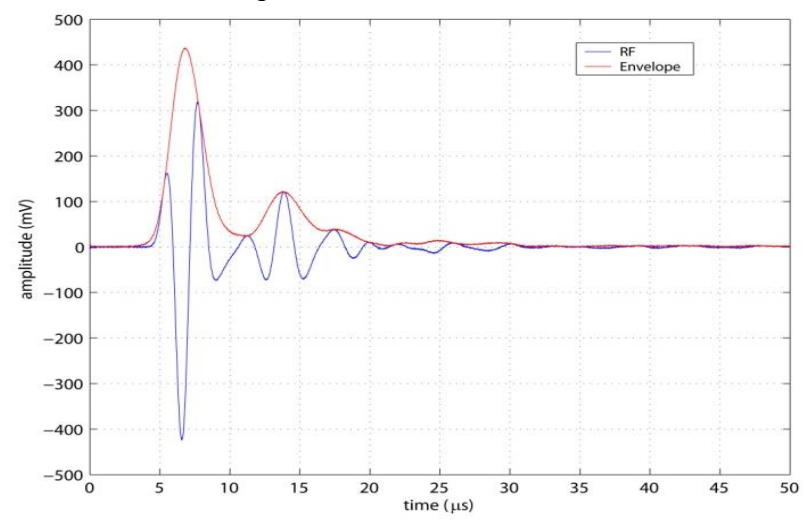

(c) Spatially-Averaged Longitudinal Waveform

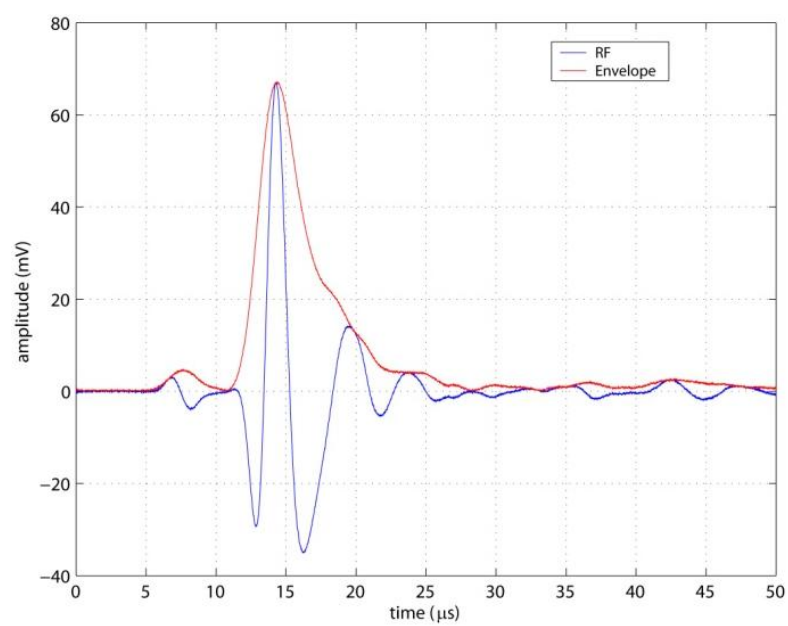

(d) Spatially-Averaged Shear Waveform
Fig. 3. Time-averaged ultrasonic waveforms obtained for the (a) longitudinal and (b) shear testing of Specimen I at $-2^{\circ} \mathrm{C}$. The spatially averaged (c) longitudinal and (d) shear waveforms

In order to obtain an accurate velocity measurement, it is necessary to know the difference in arrival times between the acquired waveforms. The highest accuracy is obtained when correlation routines are employed. This was the approach used in this work. A cross-correlation routine was written in MatLab and used to obtain the time difference between the two wave packets. The cross-correlation is defined as,

$$
R_{x y}(\tau)=\frac{1}{T} \int_{0}^{T} x(t) y(\tau+t) d t
$$

where $T$ is the period of the signals $x$ and $y$ being correlated. Effectively it is a comparison function which compares the waveforms at every possible time combination with the actual time difference taken to be at the point of highest correlation, or largest $R_{x y}$ value. Figure (4) shows the waveforms obtained for the two different specimen thicknesses of Specimen I along with the cross-correlated results.
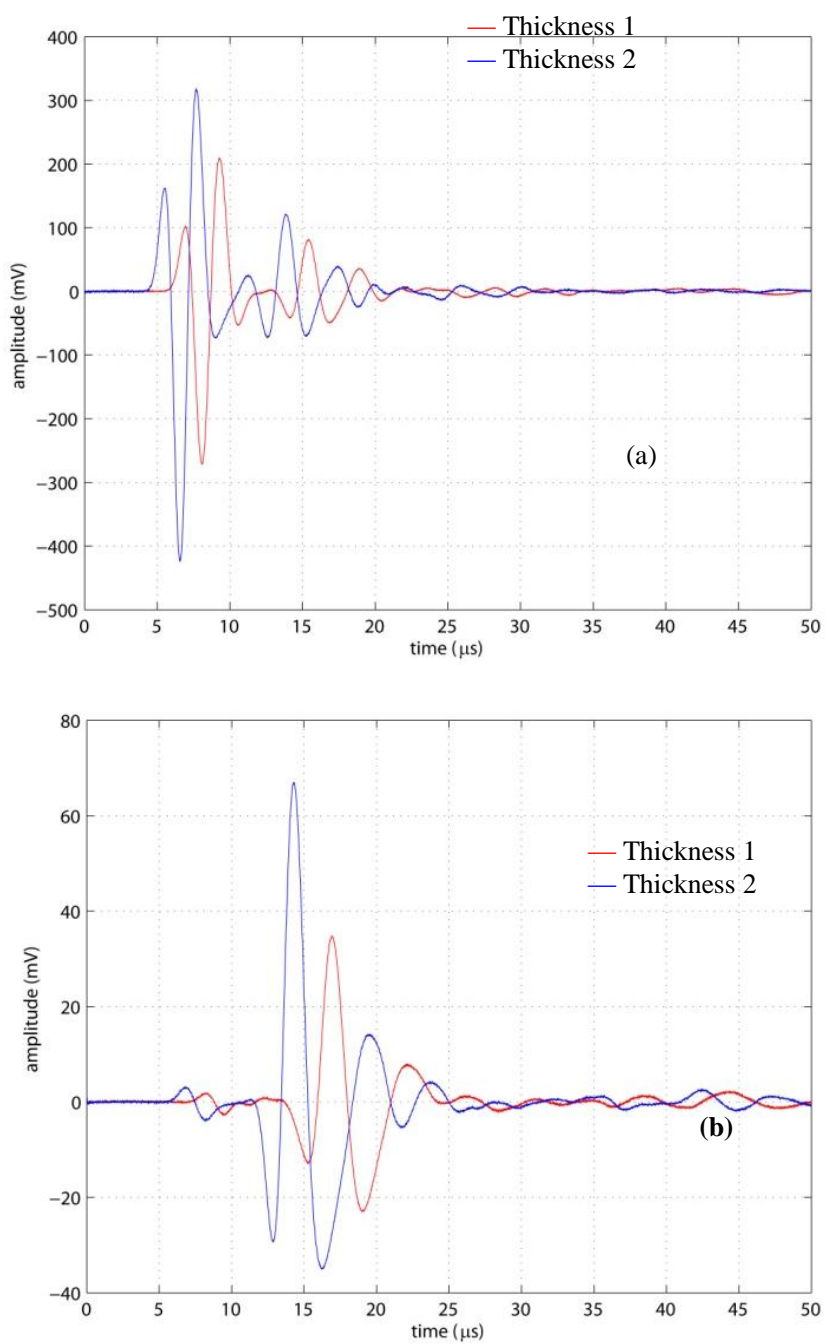

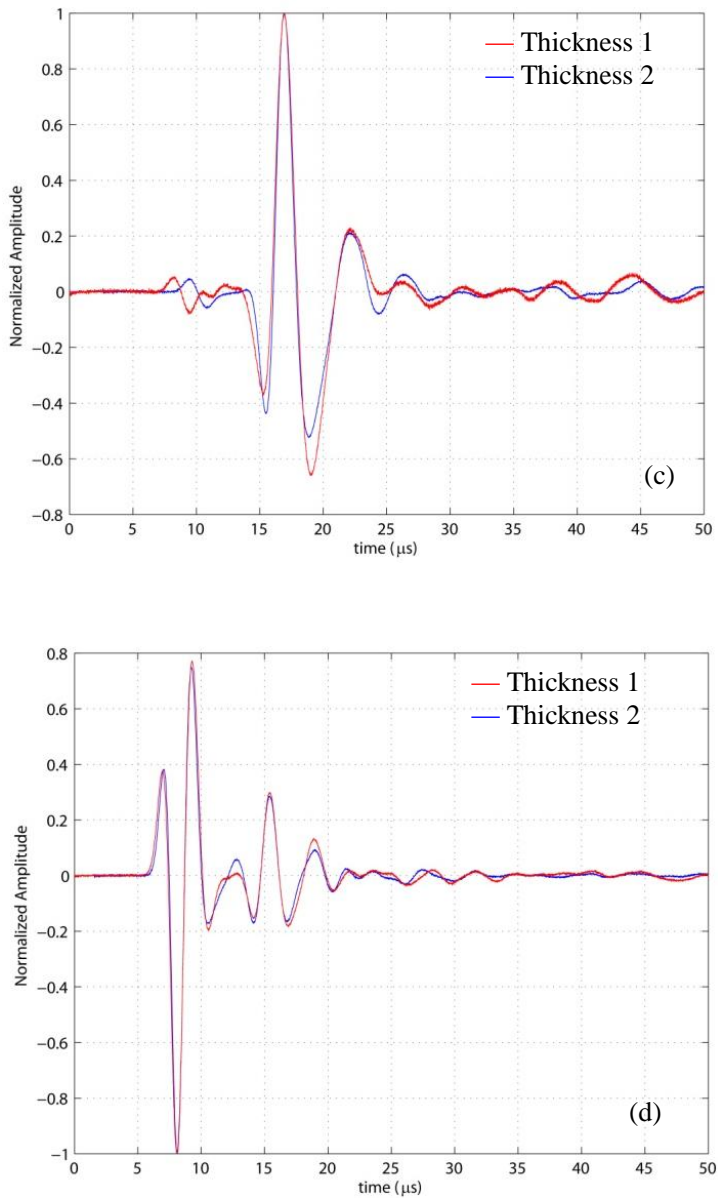

Fig. 4. Spatially averaged ultrasonic waveforms obtained for the (a) longitudinal and (b) shear testing of Specimen I at $-2^{\circ} \mathrm{C}$ and the crosscorrelated (c) longitudinal and (d) shear waveforms.

The time shift corresponding to the point of highest correlation for the longitudinal and shear data shown in Fig. (4) was $1.54 \square s$ and $2.63 \square \mathrm{s}$ at $R_{x y} \max$, respectively. Using the known difference in specimen thickness and the measured change in the time-of-flight, the corresponding wave velocity in the AC specimen can be determined from,

$c_{L, S}=\frac{h_{2}-h_{1}}{\Delta t}$

\section{Measurement of Attenuation Constant}

With the wave velocities determined, the other parameters needed to calculate the complex moduli are the longitudinal and shear wave attenuation constants. To examine how these constants might be measured experimentally, consider again the solution of the complex one-dimensional wave equation,

$$
u(x, t)=U_{0} e^{-\alpha x} e^{i k^{\prime}\left(x-c_{l} t\right)}
$$

Assuming there are two waves that have propagated distances $x_{1}$ and $x_{2}$, respectively, the amplitudes of these two waves would be given by,

$$
A_{1}=U_{0} e^{-\alpha x_{1}}
$$

$$
A_{2}=U_{0} e^{-\alpha x_{2}}
$$

Taking the ratio of Eq. (18) and Eq. (19), a convenient expression results,

$$
\frac{A_{2}}{A_{1}}=e^{-\alpha\left(x_{2}-x_{1}\right)}
$$

Again, an experiment can be formulated in which an AC sample of known thickness (or known difference in thickness between two samples) and corresponding propagated wave amplitude can be used to calculate the attenuation constant, $\alpha$. Conveniently, all of this information is contained within the data that was taken during the wave velocity measurement procedure.

If the attenuation constant is calculated using the time domain information, the results will be valid only for the center frequency of the transducer. However, if the ratio of the Fourier transformed signals is used, attenuation data will be available over the entire bandwidth of the transducer. Since the Fourier transform is a linear transform, the amplitude ratio will be conserved and the time-domain amplitudes in Eq. (20) can be replaced by the amplitudes of the Fourier transforms in the frequency domain. The definition of the Fourier transform is,

$$
\tilde{u}(\quad)=u(t) e^{i 2 t} d t
$$

and Eq. (20) is then rewritten as,

$$
\left.\frac{\left|\tilde{A}_{2}\right|}{\left|\tilde{A_{1}}\right|}=e^{\left(x_{2}\right.} x_{1}\right)
$$

where $\left|\tilde{A}_{1}\right|$ and $\left|\tilde{A}_{2}\right|$ are the amplitudes of the Fourier transforms. An expression for the attenuation constant can then be written as,

$$
(\quad)=\frac{1}{x_{2} \quad x_{1}} \ln \frac{\left|\tilde{A}_{2}\right|}{\left|\tilde{A}_{1}\right|}
$$

Figure (5) shows a plot of the Fourier transform amplitudes of the time-domain waveforms seen in plot (a) and (b) of Fig. (4). Using these transform amplitudes and an $x_{2}$ and $x_{1}$ equivalent to $h_{2}$ and $h_{1}$, respectively, a plot of the longitudinal and shear attenuation versus frequency can be generated, as seen in Fig. (6). 
The slope of the linear trend in Fig. (6) is the parameter of interest. After converting to circular frequency, this slope represents the $\alpha / \omega$ ratio that is used in the calculation of the complex elastic moduli. The attenuation information is valid over the frequency range of interest in this study.

With knowledge of the measured acoustic properties it is only left to calculate the complex moduli using Eq. (12) through Eq. (14). Table (1) and Table (2) summarize the measured acoustic properties of the two AC specimens used in this study.
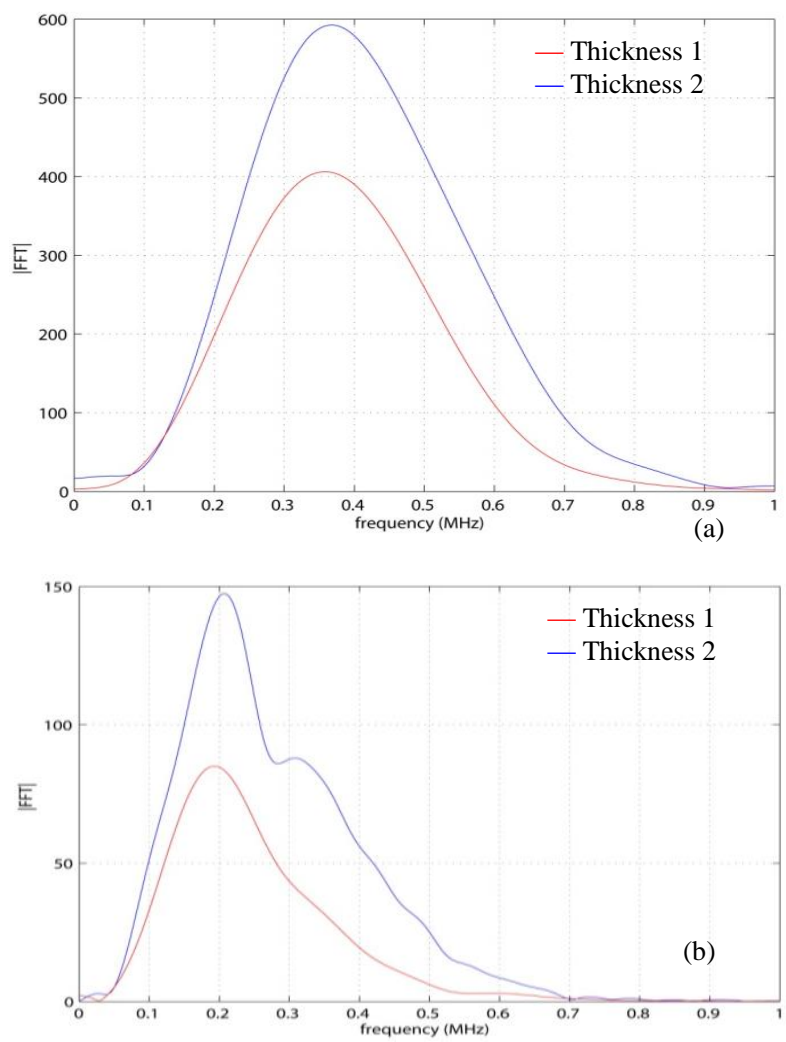

Fig. 5 Fast Fourier Transform (FFT) amplitudes of the spatially averaged (a) longitudinal and (b) shear signals obtained from Specimen I at $-2^{\circ} \mathrm{C}$.

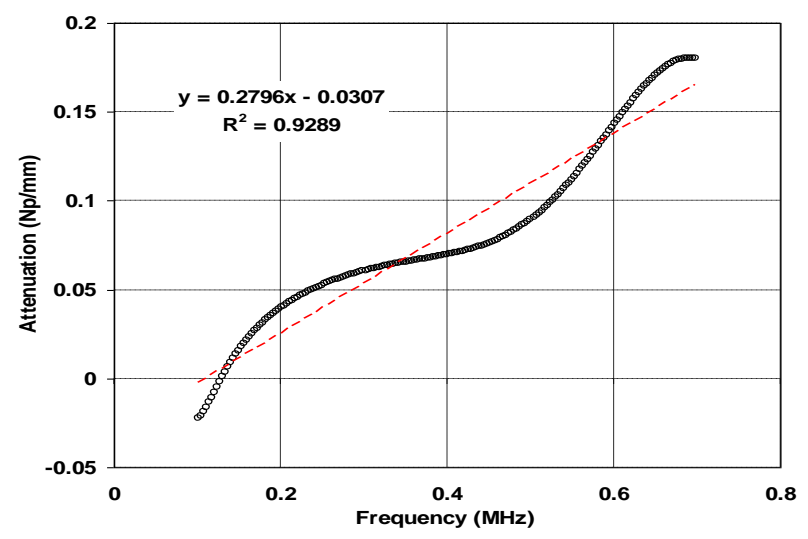

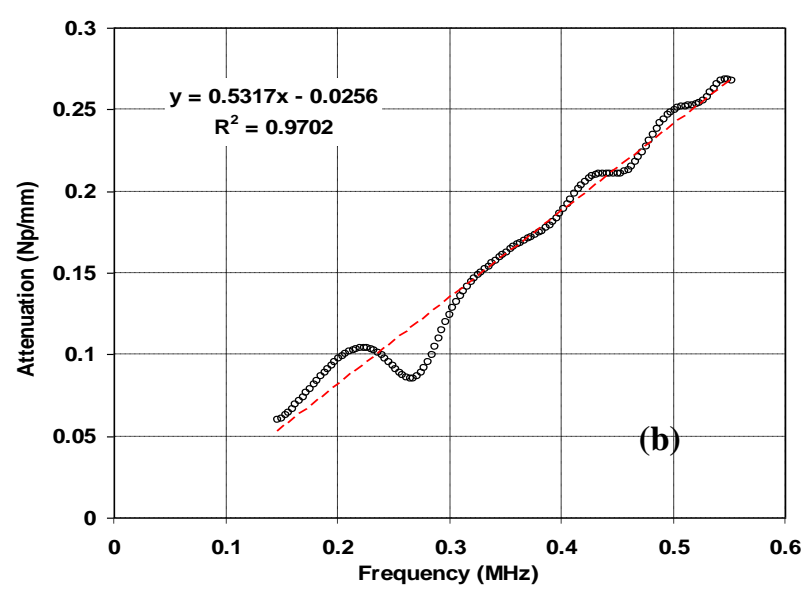

Fig. 6(a). Longitudinal and (b) shear attenuation versus frequency trend for Specimen $\mathrm{I}$ at $-2^{\circ} \mathrm{C}$. The red line is a linear fit to the data, represented by the black circles.

Table 1. Tabular summary of ultrasonic results for Specimen I at all testing temperatures

\begin{tabular}{cccccc}
\multicolumn{7}{c}{ Specimen 1 } \\
\hline Temperature & CL & CS & $\rho$ & $\alpha_{\mathrm{L}} / \omega$ & $\alpha_{\mathrm{S}} / \omega$ \\
\hline-20 & 3.44 & 2.12 & 2.36 & 0.07 & 0.05 \\
-10 & 3.91 & 2.39 & 2.36 & 0.04 & 0.01 \\
0 & 3.68 & 2.16 & 2.36 & 0.04 & 0.08 \\
10 & 3.71 & 2.10 & 2.36 & 0.02 & 0.07
\end{tabular}

Table 2. Tabular summary of ultrasonic results for Specimen II at all testing temperatures

\begin{tabular}{cccccc}
\multicolumn{7}{c}{ Specimen 2 } \\
\hline Temperature & CL & CS & $\rho$ & $\alpha_{\mathrm{L}} / \omega$ & $\alpha_{\mathrm{S}} / \omega$ \\
\hline-20 & 4.13 & 2.37 & 2.40 & 0.04 & 0.04 \\
-10 & 4.26 & 2.50 & 2.40 & 0.02 & 0.04 \\
0 & 4.05 & 2.39 & 2.40 & 0.03 & 0.05 \\
10 & 3.86 & 2.44 & 2.40 & 0.04 & 0.07
\end{tabular}

\section{Results And Analysis}

Time-Temperature superposition was used to construct the dynamic modulus and dynamic shear modulus mastercurves at a reference temperature of $0^{\circ} \mathrm{C}$ and the sigmoidal function was used for fitting the curves. As the actual testing temperatures were different, the mastercurves and ultrasonic data were shifted to a reference temperature of $0^{\circ} \mathrm{C}$. The mastercurves were also extrapolated to higher frequencies to compare with data obtained from ultrasonic testing. The extrapolated data and the actual test data for the dynamic modulus and shear modulus mastercurves are shown in Fig. (7).

From the mastercurves it is observed that the ultrasonic data matches the upper portion of the dynamic modulus and dynamic shear modulus curves more accurately for Specimen I. The difference between the results from the IDT and ultrasonic tests for Specimen I and Specimen II is 7\% and $22 \%$, respectively, for the dynamic modulus and $5 \%$ and $18 \%$, respectively, for the dynamic shear modulus. The larger variation seen for Specimen II may be due to increased wave scattering from the higher air-void population. 
The phase angle mastercurve was developed by manually shifting the vertical phase angle to compare with the ultrasonic data. It is seen from Fig. (8) that the phase angle predicted from ultrasonic measurement was higher than predicted from dynamic modulus testing. This phenomenon is somewhat expected as it was assumed that all ultrasonic attenuation is due to material dissipation. In fact, because the wavelengths in the ultrasonic region are comparable to the nominal aggregate and air void size, there will be a notable amount of wave scattering from the aggregates and air voids. When wave energy is scattered, it takes more time to reach the receiving transducer (if it reaches it at all) because of its indirect propagation path. Therefore, scattered energy will manifest as a decrease in amplitude of the directly received wave package. Future work will need to examine the severity of scattering for different ultrasonic wavelengths and aggregate and air void sizes. A compensation technique can be developed to account for energy lost in scattering and will result in a more accurate phase angle measurement.

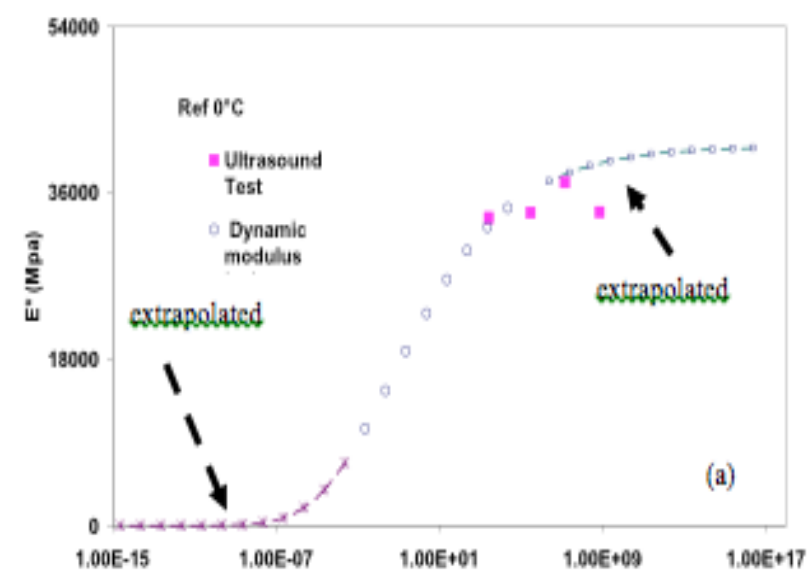

Reduced Frequency (ha)

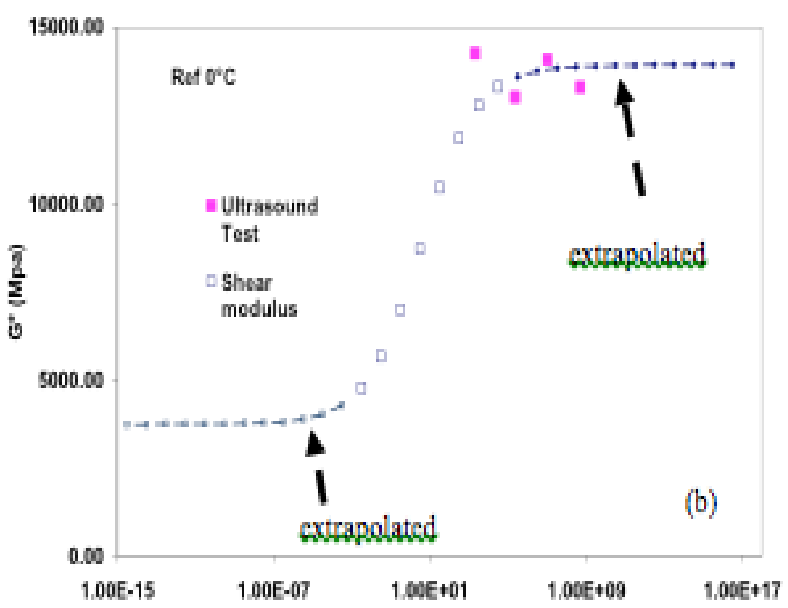

Reduced Frequency (he)
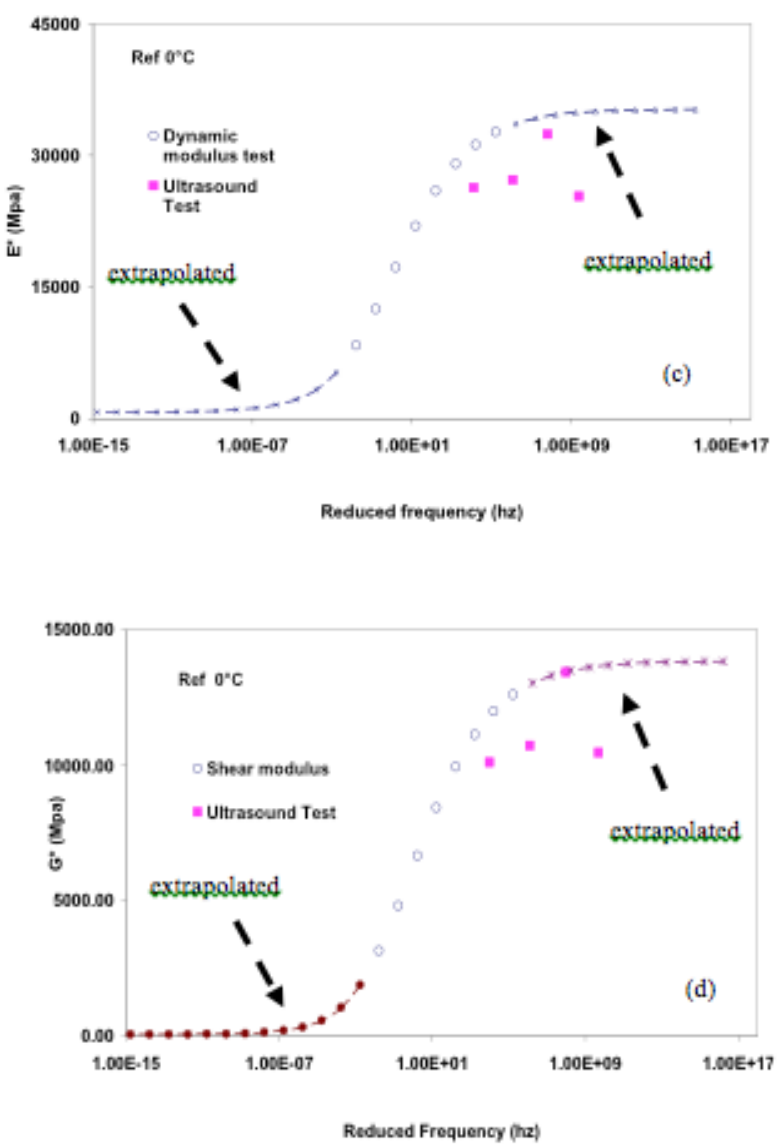

Fig. 7. Comparison of ultrasonic data with (a) dynamic modulus and (b) dynamic shear modulus for Specimen I and of (c) dynamic modulus and (d) dynamic shear modulus of Specimen II.
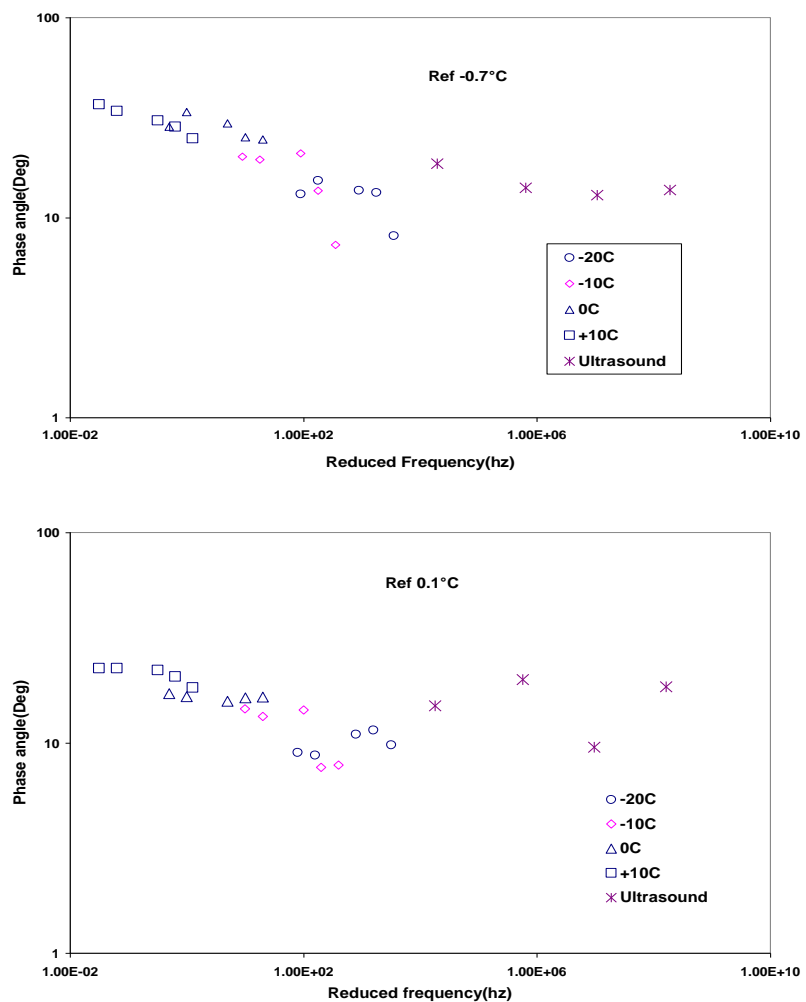

Fig. 8. Comparison of phase angle for: (a) Specimen I; (b) Specimen II. 9. Conclusion 
In this study, a new ultrasonic method was utilized to predict the complex moduli of AC near the upper asymptote of the dynamic modulus and dynamic shear modulus mastercurves. A theoretical demonstration of how ultrasonic measurements could be used to calculate complex moduli was presented. Two specimens were tested using the ultrasonic method and the IDT dynamic modulus method. The dynamic modulus, dynamic shear modulus, and phase angle mastercurves were constructed using the IDT test data and the upper asymptotes of the mastercurves were extrapolated to compare with the results from the longitudinal- and shear-wave ultrasound tests. It was found that the upper asymptote of the dynamic modulus and dynamic shear modulus mastercurves matched well with the ultrasonic prediction for the AC specimen with lower air-void content. The phase angle data obtained from ultrasound tests were higher than the dynamic modulus tests in both specimens. This phenomenon may be related to increased energy loss due to wave scattering from air voids and aggregates.

Ultrasonic equipment is relatively light and portable, making the possibility of field testing a reality. This has been the primary motivation of this work. Future work will concentrate on characterizing the relationship between airvoid content, aggregate gradation, asphalt binder content, wave propagation, and wave scattering. If understood properly, a compensation factor could be developed to account for wave scattering and, in turn, improve the accuracy of the phase angle measurement. More AC replicates will be tested using the ultrasonic method and compared to the results of traditional dynamic modulus tests. This should be done using additional types of mixtures since only one mixture was used in this study.

\section{References}

1. Kweon, G. and Y. R. Kim. Determination of Asphalt Concrete Complex Modulus with Impact Resonance Test. Transportation Research Record: Journal of the Transportation Research Board, No. 1970, Transportation Research Board of the National Academies, Washington, D.C., 2006, pp. 151-160.

2. Sztukiewicz, R. J. Application of ultrasonic methods in asphalt concrete characterization. Ultrasonics, Vol. 1991(29), 1989, pp. 512.

3. Norambuena-Contreras, J, Castro-Fresno, D, Vega-Zamanillo, A, Celaya, M, and Lombillo-Vozmediano, I. (2010). Dynamic modulus of asphalt mixture by ultrasonic direct test. NDT E Int., 43(7), 629-634, 2010

4. Pellinen, T. K. and M. W. Witczak. Use of Stiffness of Hot-Mix Asphalt as a Simple Performance Test. Transportation Research Record: Journal of the Transportation Research Board, No. 1789, Transportation Research Board of the National Academies, Washington, D.C., 2002, pp. 80-90.

5. Witczak MW, Kaloush K, Pellinen T, El-Basyouny M, Von Quintus H. Simple Performance Test for Superpave Mix Design, Research Report FHWA 465. Washington DC; 2002.

6. Chehab, G.R., Y.R. Kim, R.A. Schapery, M. Witczack, R. Bonaquist, 2003, "Characterization of Asphalt Concrete in Uniaxial
Tension Using a Viscoelastoplastic Model," Journal of Asphalt Paving Technology Association of Asphalt Pavement Technologists; Vol. 72.

7. Momen, M. Complex Modulus Determination of Asphalt Concrete using the Indirect Tension Test. M.S. Thesis, North Carolina State University, Raleigh, NC, 2004.

8. Chehab, G., Y. R. Kim, R. A. Schapery, M. Witczack, and R. Bonaquist. Time-Temperature Superposition Principle for Asphalt Concrete Mixtures with Growing Damage in Tension. Asphalt Paving Technology, AAPT, Vol. 71, 2002, pp. 559-593.

9. Read, W. T. Stress Analysis for Compressible Viscoelastic Materials. Journal of Applied Physics, Vol. 21, 1950, pp. 671-674.

10. Christensen, R.M. Theory of viscoelasticity: an introduction. Academic Press, New York, NY, 1981.

11. Haddad, Y.M. Viscoelasticity of engineering materials. Chapman \& Hall, London, U.K., 1995.

12. Barshinger, J. N. and J. L. Rose. Guided Wave Propagation in an Elastic Hollow Cylinder Coated with a Viscoelastic Material. IEEE Transactions on Ultrasonics, Ferroelectrics, and Frequency Control, Vol. 51(11), 2004, pp. 1547-1556. 